\title{
Realistic shell model description of beta decay
}

\author{
Angela Gargano ${ }^{1, *}$, Luigi Coraggio ${ }^{1}$, Luca De Angelis ${ }^{1}$, Tokuro Fukui ${ }^{1}$, and Nunzio Itaco $^{1,2}$ \\ ${ }^{1}$ Istituto Nazionale di Fisica Nucleare, Complesso Universitario di Monte S. Angelo, Via Cintia - I-80126 Napoli, Italy \\ ${ }^{2}$ Dipartimento di Matematica e Fisica, Università degli Studi della Campania "Luigi Vanvitelli", viale Abramo Lincoln 5 - I-81100 \\ Caserta, Italy
}

\begin{abstract}
In this paper, we extend our previous realistic shell-model study of Gamow-Teller strengths and double- $\beta$ decay properties for nuclei around ${ }^{132} \mathrm{Sn}$ to lighter mass nuclei. The effective shell-model Hamiltonian and transition operators are microscopically derived by way of many-body perturbation theory, without resorting to empirical parameters. Our main aim is to further check the reliability of our approach and verify its stability in other mass regions. Calculated energy spectra as well as electric-quadrupole and $\beta$-decay properties are presented for ${ }^{76} \mathrm{Ge}$ and ${ }^{82} \mathrm{Se}$ and compared with the experimental data, together with some results for ${ }^{130} \mathrm{Te}$ and ${ }^{136} \mathrm{Xe}$ already reported in our previous paper. Finally, some preliminary results of nuclear matrix elements responsible for the neutrinoless double beta decay, calculated by using the bare operator, are shown.
\end{abstract}

\section{Introduction}

The neutrinoless double beta $(0 v \beta \beta)$ decay provides a major opportunity to address relevant questions about the mass and the nature of neutrinos. Its study is on top of the agenda of current experimental and theoretical research programs around the world [1]. The estimate of the associated lifetimes, as well as the extraction of the neutrino effective mass once a decay signal has been observed, requires the knowledge of the matrix elements of the transition operators between the parent and grand-daughter nuclei.

The calculation of nuclear matrix elements (NMEs) responsible for the $0 v \beta \beta$ decay has been tackled in the framework of several theoretical approaches, as the quasiparticle random-phase approximation, the interacting boson model, the shell model, and energy density functional methods (see, for instance, [2-6] and references therein). However, the spread of the values obtained with different approaches is quite large. As a matter of fact, results, obtained with different nuclear structure models, largely differ, thus indicating the need to improve the accuracy of nuclear structure calculations.

By analogy with single $-\beta$ and two-neutrino double $-\beta$ $(2 v \beta \beta)$ processes whose predicted lifetimes are almost always shorter than measured lifetimes, a quenching of the axial coupling constant $g_{A}$ is quite often introduced in order to take into account missing many-body correlations and/or coupling with non-nucleonic degrees of freedom in a phenomenological way. A review of the theoretical methods and calculations employed to predict the NMEs of the $0 v \beta \beta$ decay is given in the recent review paper of Ref. [7], where the renormalization of the axial vector coupling $g_{A}$ is also discussed in detail.

\footnotetext{
*e-mail: gargano@na.infn.it
}

A valuable tool to address this problem is given by the realistic shell model (SM) $[8,9]$. Within this approach, the SM Hamiltonian and the transition operators are derived from a realistic free nuclear potential by way of manybody perturbation theory on the same footing. In this way, one can account for renormalization effects induced by missing correlations in the nuclear wave functions as resulting from truncations of the Hilbert space.

As a first step towards the calculation of the NMEs of the $0 v \beta \beta$ decay, we have recently performed a realistic SM study of ${ }^{130} \mathrm{Te}$ and ${ }^{136} \mathrm{Xe}[10]$, which are candidates for the observation of the $0 v \beta \beta$ decay. The focus was on GamowTeller (GT) strengths and $2 v \beta \beta$ decay NMEs, which may provide a relevant test of our approach. It turned out that the available experimental data - energy spectra, electricquadrupole transitions and beta decay properties - are well described by our calculations without the need to resort to empirical parameters, and, in particular, without quenching the $g_{A}$ constant.

To further check the reliability of our approach and investigate the renormalization effects in different mass regions, we have carried out realistic SM calculations for other nuclei, which are also candidates for the observation of the $0 v \beta \beta$ decay. Results will be presented in a forthcoming paper [11]. Here, we present energy spectra as well as electric-quadrupole transitions and beta decay properties for ${ }^{76} \mathrm{Ge}$ and ${ }^{82} \mathrm{Se}$. For the sake of completeness, we also show the GT strengths and $2 v \beta \beta$ NMEs of ${ }^{130} \mathrm{Te}$ and ${ }^{136} \mathrm{Xe}$.

Our starting point is again the high-precision $\mathrm{CD}$ Bonn nucleon-nucleon $(N N)$ potential [12], whose repulsive high-momentum components are smoothed out using the $V_{\text {low-k }}$ approach [13]. Then, the effective SM Hamiltonian and transition operators are derived within the timedependent perturbation theory [14], including diagrams up to the third order. 
Within this approach we have also computed the $0 v \beta \beta$ decay NMEs by employing the bare operators for all the four nuclei under investigation. A comparison of the obtained NMEs results with those of other SM calculations may provide an important test of our wave functions, before calculating the NMEs with effective operators.

The paper is organized as follows. In Sec. 2, we outline the theoretical framework of our approach and give some details about the derivation of the SM Hamiltonian and effective transition operators. Section 3 is devoted to the presentation of the results of our calculations and to the comparison with the available experimental data. A summary and some concluding remarks are given in the last section.

\section{Theoretical framework}

In our realistic SM calculations for ${ }^{76} \mathrm{Ge}$ and ${ }^{82} \mathrm{Se}$, we have taken ${ }^{56} \mathrm{Ni}$ as closed core and employed a model space spanned by the four orbitals $0 f_{5 / 2}, 1 p_{3 / 2}, 1 p_{1 / 2}, 0 g_{9 / 2}$ for both protons and neutrons. For ${ }^{130} \mathrm{Te}$ and ${ }^{136} \mathrm{Xe}$, we have assumed that valence protons and neutrons occupy the five orbitals $0 g_{7 / 2}, 1 d_{5 / 2}, 1 d_{3 / 2}, 2 s_{1 / 2}, 0 h_{11 / 2}$ outside ${ }^{100} \mathrm{Sn}$. In both cases, the effective Hamiltonian and transition operators have been derived within the framework of the manybody perturbation theory outlined below.

Starting from the Hamiltonian, $H$, for a system of $A$ nucleons interacting via two-body forces, we introduce an auxiliary potential so that it can be written as the sum of a one-body component $H_{0}$, which describes the independent motion of the nucleons, and a residual interaction $H_{1}$.

$$
H=(T+U)+\left(V_{\mathrm{NN}}-U\right)=H_{0}+H_{1},
$$

where $T$ is the kinetic energy and $V_{\mathrm{NN}}$ a low-momentum two-body potential, obtained through the $V_{\text {low-k }}$ procedure [13] from the CD-Bonn $N N$ potential [12], by using a cutoff momentum $\Lambda=2.6 \mathrm{fm}^{-1}$. The harmonic oscillator potential is chosen for $U$ and the Coulomb force added to the $V_{\text {low-k }}$ for protons.

Then, the diagonalization of the Hamiltonian (1) in the full Hilbert space is reduced to a model-space eigenvalue problem introducing an effective Hamiltonian, $H_{\text {eff }}$, acting only on the truncated model space defined in terms of the eigenvectors of $H_{0}$.

A well-established approach to the determination of the effective Hamiltonian is given by the Kuo-Lee-Ratcliff folded-diagram expansion [14]. A detailed description of this approach can be found in Refs. [8, 9]. Here, we would like to highlight only that it is based on the perturbative expansion of the so-called $\hat{Q}$-box

$$
\hat{Q}(\epsilon)=P H_{1} P+P H_{1} Q \frac{1}{\epsilon-Q H Q} Q H_{1} P,
$$

where $P$ and $Q$ are projection operators onto the model space and its complement.

The $\hat{Q}$-box is calculated including one- and two-body Goldstone diagrams through third order in $H_{1}$. Calculations beyond the third order in perturbation theory are computationally prohibitive, so we have computed also the Padé approximant [2|1] of the $\hat{Q}$-box to obtain a value to which the perturbation series should converge, as suggested in [15].

The effective Hamiltonian is written as a sum of products of the $\hat{Q}$-box and its derivatives with respect to the unperturbed energy. The sum is carried out by means of the Lee-Suzuki iterative technique [16].

It is worth noting that our effective Hamiltonian is derived for a two-valence-particle nucleus. Therefore, it contains one- and two-body components, the former, summed to the eigenvalues of $H_{0}$, gives the theoretical singleparticle energies.

Concerning the effective transition operators, they have been derived within the same framework of and consistently with the SM Hamiltonian, employing the approach proposed by Suzuki and Okamoto in Ref. [17]. In this context, similarly to the $\hat{Q}$-box, a $\hat{X}$-box is introduced that we have calculated including diagrams up to third order in the perturbation theory (see Ref. [10]).

Using this procedure, we have calculated the effective matrix elements of one-body operators, as it is the case of the electric-quadrupole (E2) and GT operators, for a single particle beyond ${ }^{56} \mathrm{Ni}$ and ${ }^{100} \mathrm{Sn}$. These effective matrix elements have been used to compute the $B(E 2)$ and GT strengths, as well as the NMEs of the $2 v \beta \beta$ decay reported in Sec. 3.

Table 1. Bare and effective proton-neutron matrix elements for the model space outside ${ }^{56} \mathrm{Ni}$. In the last column the corresponding quenching factors are reported (see text for details).

\begin{tabular}{llrrc}
\hline$n_{a} l_{a} j_{a}$ & $n_{b} l_{b} j_{b}$ & Bare & Effective & quenching factor \\
\hline $0 f_{5 / 2}$ & $0 f_{5 / 2}$ & -2.69 & -0.98 & 0.36 \\
$0 f_{5 / 2}$ & $1 p_{3 / 2}$ & 0 & -0.14 & \\
$1 p_{3 / 2}$ & $0 f_{5 / 2}$ & 0 & 0.05 & \\
$1 p_{3 / 2}$ & $1 p_{3 / 2}$ & 3.27 & 2.03 & 0.62 \\
$1 p_{3 / 2}$ & $1 p_{1 / 2}$ & -2.95 & -1.62 & 0.55 \\
$1 p_{1 / 2}$ & $1 p_{3 / 2}$ & 2.95 & 1.71 & 0.58 \\
$1 p_{1 / 2}$ & $1 p_{1 / 2}$ & -1.04 & -0.70 & 0.67 \\
$0 g_{9 / 2}$ & $0 g_{9 / 2}$ & 4.47 & 3.13 & 0.70 \\
\hline
\end{tabular}

Table 2. Bare and effective proton-neutron matrix elements for the model space outside ${ }^{100} \mathrm{Sn}$. In the last column the corresponding quenching factors are reported (see text for details).

\begin{tabular}{llrrc}
\hline$n_{a} l_{a} j_{a}$ & $n_{b} l_{b} j_{b}$ & Bare & Effective & quenching factor \\
\hline $0 g_{7 / 2}$ & $0 g_{7 / 2}$ & -2.48 & -1.24 & 0.50 \\
$0 g_{7 / 2}$ & $1 d_{5 / 2}$ & 0 & -0.14 & \\
$1 d_{5 / 2}$ & $0 g_{7 / 2}$ & 0 & 0.02 & \\
$1 d_{5 / 2}$ & $1 d_{5 / 2}$ & 2.91 & 1.86 & 0.64 \\
$1 d_{5 / 2}$ & $1 d_{3 / 2}$ & -3.10 & -1.75 & 0.56 \\
$1 d_{3 / 2}$ & $1 d_{5 / 2}$ & 3.10 & 1.94 & 0.63 \\
$1 d_{3 / 2}$ & $1 d_{3 / 2}$ & -1.55 & -1.02 & 0.66 \\
$1 d_{3 / 2}$ & $2 s_{1 / 2}$ & 0 & -0.12 & \\
$2 s_{1 / 2}$ & $1 d_{3 / 2}$ & 0 & 0.09 & \\
$2 s_{1 / 2}$ & $2 s_{1 / 2}$ & 2.46 & 1.60 & 0.65 \\
$0 h_{11 / 2}$ & $0 h_{11 / 2}$ & 3.76 & 2.60 & 0.69 \\
\hline
\end{tabular}


In order to show the effects due to the renormalizations induced by the truncation of the Hilbert space for the $0 f_{5 / 2} 1 p 0 g_{9 / 2}$ and $0 g_{7 / 2} 1 d 2 s 0 h_{11 / 2}$ model spaces, the bare proton-neutron matrix elements of the $\mathrm{GT}^{-}$operator are compared with the effective ones in Tables 1 and 2. For the sake of clarity, we also report quenching factors, namely the ratio between the effective and bare matrix elements, except for transitions that are forbidden for the bare $\mathrm{GT}^{-}$ operator. It is worth mentioning that the values shown in Table 2 were taken from [10].

From both Tables, we see that all effective matrix elements, as expected, are reduced with respect to the bare ones, but the reduction factor depends on the initial and final states, ranging from 0.36 to 0.70 in Table 1 and from 0.50 to 0.69 in Table 2. So, microscopic renormalizations lead to selective effects and in particular make forbidden off-diagonal matrix elements different from zero, which is not possible when using an empirical quenching factor.

In Sec. 3, we also report some preliminary results of the $0 v \beta \beta$ NMEs, which have been calculated employing the bare two-body operator:

$$
O^{0 v}=O_{12}^{G T}-\left(\frac{g_{V}}{g_{A}}\right)^{2} O_{12}^{F}-O_{12}^{T},
$$

where the vector and axial constants are $g_{V}=1$ and $g_{A}=1.269$, respectively; and the GT, Fermi, and tensor components are explicitly given by

$$
\begin{aligned}
O_{12}^{G T} & =\tau_{1}^{-} \tau_{2}^{-} \vec{\sigma}_{1} \cdot \vec{\sigma}_{2} H_{G T}(r), \\
O_{12}^{F} & =\tau_{1}^{-} \tau_{2}^{-} H_{F}(r), \\
O_{12}^{T} & =\tau_{1}^{-} \tau_{2}^{-}\left[3\left(\vec{\sigma}_{1} \cdot \hat{r}\right)\left(\vec{\sigma}_{2} \cdot \hat{r}\right)-\vec{\sigma}_{1} \cdot \vec{\sigma}_{2}\right] H_{T}(r) .
\end{aligned}
$$

Here the functions $H_{\alpha}(r)$ denote the neutrino potentials.

\section{Results}

The results presented in this section have been obtained by employing the effective Hamiltonian and the transition operators derived within the theoretical framework discussed in Sec. 2. Calculations have been carried out using the shell-model code ANTOINE [18].

We start by presenting the spectra and the E2 transition rates of ${ }^{76} \mathrm{Ge}$ and ${ }^{82} \mathrm{Se}$, results for ${ }^{130} \mathrm{Te}$ and ${ }^{136} \mathrm{Xe}$ have been already reported in Ref. [10]. In figs. 1 and 2, the calculated low-energy spectra and $B(E 2)$ values are compared with the experimental data for ${ }^{76} \mathrm{Ge}[19,20]$ and ${ }^{82} \mathrm{Se}[19]$, respectively. Note that arrows are proportional to $B(E 2)$ strengths.

We see that the agreement between theory and experiment for excitation energies is quite reasonable, although not completely satisfactory. All calculated states are, in fact, shifted up by an amount ranging from about 200 to $500 \mathrm{keV}$, which may be related to the lack of the $0 f_{7 / 2}$ orbital in our model space. The experimental E2 transition rates, however, are well reproduced by our calculations, testifying the reliability of our shell-model wave functions and effective electric-quadrupole transition operator. The error, in fact, is at most $30 \%$ except for the $B\left(E 2 ; 0^{+} \rightarrow 2^{+}\right)$

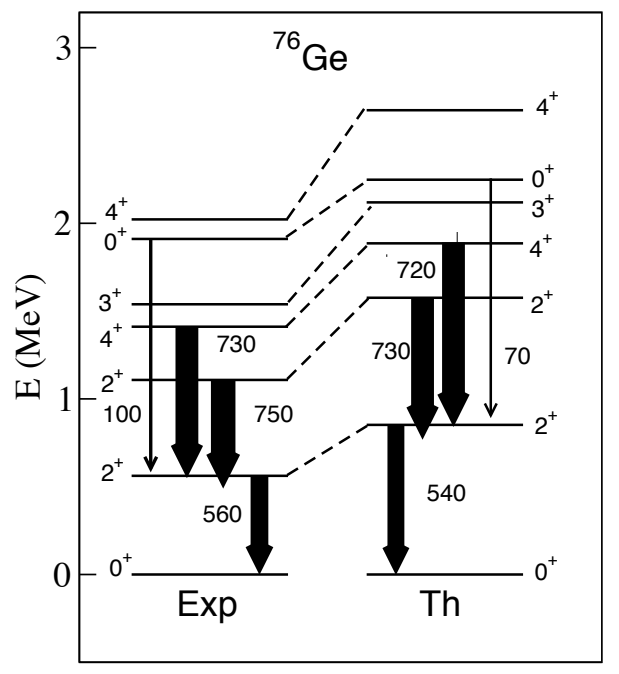

Figure 1. Comparison between theory and experiment of spectra and $B(E 2)$ strengths (in $e^{2} \mathrm{fm}^{4}$ ) in ${ }^{76} \mathrm{Ge}$.

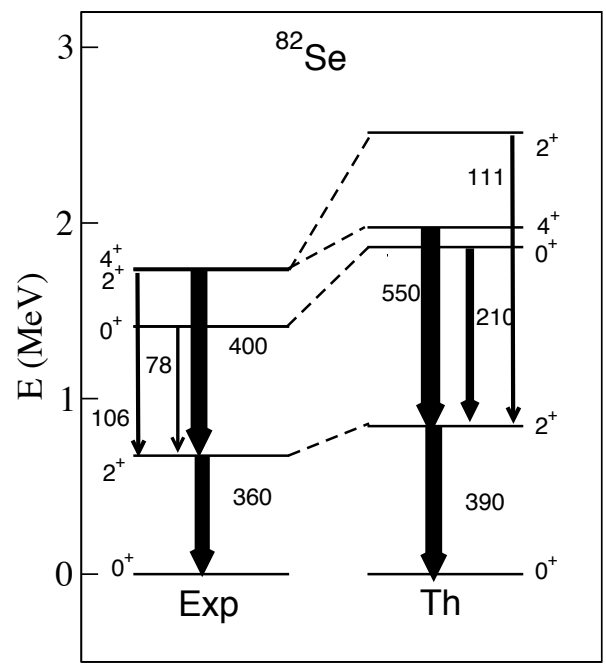

Figure 2. Comparison between theory and experiment of spectra and $B(E 2)$ strengths (in $e^{2} \mathrm{fm}^{4}$ ) in ${ }^{82} \mathrm{Se}$.

in ${ }^{82} \mathrm{Se}$. In this case, the calculated value is 3 times larger than the experimental one.

In Table 3 we report the results of the total $\mathrm{GT}^{-}$ strength, $S\left(\mathrm{GT}^{-}\right)$, that is defined as

$$
S\left(G T^{-}\right)=\sum_{f} \frac{\left|\left\langle\Phi_{f}\left\|\sum_{j} \vec{\sigma}_{j} \tau_{j}^{-}\right\| \Phi_{i}\right\rangle\right|^{2}}{2 J_{i}+1}
$$

where $\Phi_{i}$ refers to the ground state of the parent nucleus, while $\Phi_{f}$ to all the possible final states in the daughter nucleus up to a given energy.

The total $\mathrm{GT}^{-}$strengths are calculated using both the bare and effective $\mathrm{GT}^{-}$operators for ${ }^{76} \mathrm{Ge},{ }^{82} \mathrm{Se},{ }^{130} \mathrm{Te}$ and ${ }^{136} \mathrm{Xe}$. They are compared with the experimental values extracted from single charge-exchange experiments [2124]

From the inspection of Table 3 , it can be seen that while the $\mathrm{S}\left(\mathrm{GT}^{-}\right)$s calculated with the bare operators are 
Table 3. Calculated and experimental total $\mathrm{GT}^{-}$strengths up to $3 \mathrm{MeV}$ excitation energy for ${ }^{76} \mathrm{Ge},{ }^{82} \mathrm{Se},{ }^{130} \mathrm{Te}$, and up to 4.5 $\mathrm{MeV}$ for ${ }^{136} \mathrm{Xe}$ (see text for details).

\begin{tabular}{lcrc}
\hline & Bare & Effective & Experimental \\
\hline${ }^{76} \mathrm{Ge}$ & 2.98 & 1.06 & $1.02 \pm 0.16$ \\
${ }^{82} \mathrm{Se}$ & 2.49 & 0.79 & $0.83 \pm 0.10$ \\
${ }^{130} \mathrm{Te}$ & 2.55 & 0.84 & $0.75 \pm 0.05$ \\
${ }^{136} \mathrm{Xe}$ & 3.21 & 0.94 & $1.33 \pm 0.07$ \\
\hline
\end{tabular}

significantly larger that the experimental ones, a quite good agreement between theory and experiment is obtained when using the effective operators. This underlines the crucial role played by the many-body renormalization, that, as shown in Table 1, leads to a strong reduction of the bare matrix elements of the $\mathrm{GT}^{-}$operator. As a matter of fact, for all the nuclei under investigation the theoretical total $\mathrm{GT}^{-}$strengths are reduced by a factor around 3 .

As regards the $2 v \beta \beta$ decay we have calculated the NMEs, as defined by the following expression

$$
M_{2 v}^{G T}=\sum_{n} \frac{\left\langle 0_{f}^{+}\left\|\vec{\sigma} \tau^{-}\right\| 1_{n}^{+}\right\rangle\left\langle 1_{n}^{+}\left\|\vec{\sigma} \tau^{-}\right\| 0_{i}^{+}\right\rangle}{E_{n}-\left(M_{i}-M_{f}\right) / 2},
$$

where $E_{n}$ is the excitation energy of the $n$-th $J^{\pi}=1^{+}$state, $E_{0}=\frac{1}{2} Q_{\beta \beta}+\Delta M, Q_{\beta \beta}$ being the $Q$-value corresponding to the $\beta \beta$ decay, and $\Delta M$ the mass difference between the parent and intermediate nuclei.

In fig. 3, we report a correlation plot between the experimental and calculated NME values. The experimental data have been taken from Ref. [25], where they are calculated from the measured half-life values for the $2 v \beta \beta$ decay, $T_{1 / 2}^{2 v}$, using the relation

$$
\left[T_{1 / 2}^{2 v}\right]^{-1}=G^{2 v}\left[M_{2 v}^{G T}\right]^{2},
$$

The NMEs calculated with the bare GT operator are reported as blue dots, and those obtained employing the effective one with black triangles.

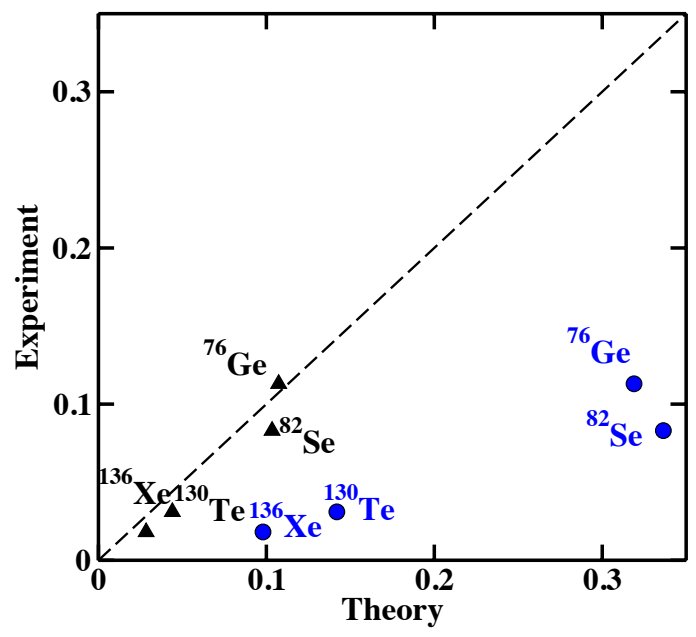

Figure 3. Correlation plot between experimental and calculated $2 v \beta \beta$ NMEs (see text for details).
As can be seen, the position of the blue dots evidences that the NMEs calculated with the bare GT operator are largely overestimated with respect to the experimental ones, while the fundamental role played by the effective GT operator is supported by the fact that the black triangles lie very close to the bisector of the correlation plot.

Finally, in fig. 4 we present our results for the $0 v \beta \beta$ decay NMEs, obtained employing the bare operator. The calculation of the effective two-body operator is under way, and the results will be the subject of a forthcoming paper [26]. Our theoretical NMEs for ${ }^{76} \mathrm{Ge},{ }^{82} \mathrm{Se},{ }^{130} \mathrm{Te}$, and ${ }^{136} \mathrm{Xe}$ (black dots), calculated summing only the GT and Fermi contributions, are compared with those obtained in other SM calculations, that have employed the same model space and $0 v \beta \beta$ decay operator, but different SM Hamiltonians. More precisely, we refer to the results of the Strasbourg-Madrid group (blue dots) [27], and those of Horoi and coworkers (red dots) [28, 29].

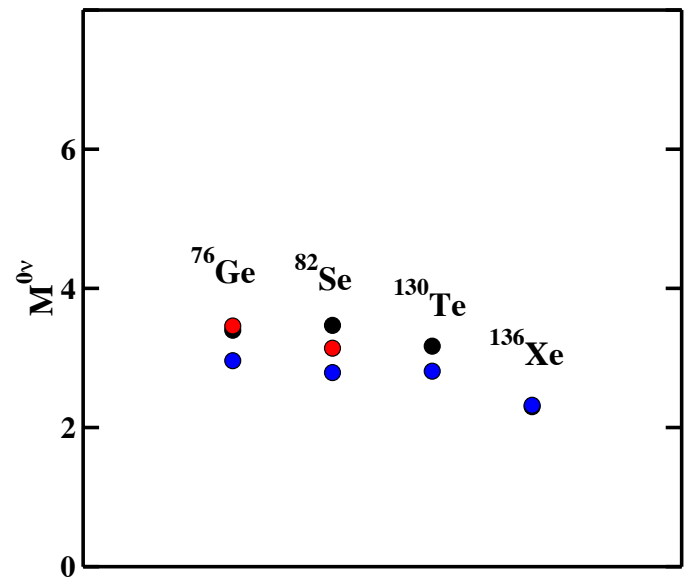

Figure 4. $0 v \beta \beta$ NMEs calculated with different SM calculations, including present ones (see text for details).

From the inspection of fig. 4, it can be observed that all SM calculations, for all nuclei under investigation, provide NME values very close to each other, notwithstanding the different employed Hamiltonians. This outcome can be considered as a benchmark for the SM eigenfunctions, and supports the SM approach to predict reliable $0 v \beta \beta$ NMEs.

\section{Summary and Perspectives}

In the present paper, we have presented the results of realistic SM calculations for ${ }^{76} \mathrm{Ge},{ }^{82} \mathrm{Se},{ }^{130} \mathrm{Te}$, and ${ }^{136} \mathrm{Xe}$, with special attention to their GT decay properties.

In these calculations, the SM Hamiltonian, including both the two-body matrix elements and single-particle energies, as well as the transition operators are calculated by way of the many-body perturbation theory, starting from a low-momentum interaction derived from the highprecision CD-Bonn free $N N$ potential. This means that renormalization effects due to the reduction of the Hilbert space are included in our theoretical effective operators, without resorting of any empirical parameters. 
The results report here are part of a study - started with our previous work on nuclei around ${ }^{132} \mathrm{Sn}[10]$ - we have undertaken with the main aim to test our approach to the calculation of the $0 v \beta \beta$ decay NME.

The calculated energy spectra, electric-quadrupole transitions, GT strengths and NMEs of the $2 v \beta \beta$ decay are in good agreement with experimental data and the quality of the results is very similar for all the four nuclei under investigation.

This opens up good prospects to a fully microscopic calculation of $0 v \beta \beta$ decay NME. In this connection, we have reported results of this matrix element for ${ }^{76} \mathrm{Ge},{ }^{82} \mathrm{Se}$, ${ }^{130} \mathrm{Te}$, and ${ }^{136} \mathrm{Xe}$, by using the bare $0 v \beta \beta$ decay operator and including only its GT and Fermi components. We have found that our matrix elements are very close to those obtained in other SM calculations, that employ the same model space and $0 v \beta \beta$ decay operator, but different SM Hamiltonians. This may be seen as another proof of the reliability of our wave functions.

Our next step towards the calculation of the $0 v \beta \beta$ decay NME is the derivation of the effective operator within the theoretical framework described in Sec. 3, by considering also the tensor component. Then, there also other effects that we plan to include in the renormalization of the decay operators, as, for instance, the contributions of three-body correlations among the valence nucleons, the so-called blocking effect, and those arising from the quark structure of nucleons. As mentioned above, work along these lines is under way.

\section{References}

[1] O. Cremonesi and M. Pavan, Adv. High Energy Physics 2014, 951432 (2014)

[2] J. D. Vergados, H. Ejiri H and F. Šimkovic, Rep. Prog. Phys. 75, 106301 (2012)

[3] J. Barea, J. Kotila, and F. Iachello, Phys. Rev. C 91, 034304 (2015)

[4] F. Šimkovic, A. Faessler, V. Rodin, and P. Vogel, Phys. Rev. C 87, 045501 (2013)

[5] J. Menéndez, T. R. Rodríguez, G. Martínez-Pinedo, and A. Poves, Phys. Rev. C 90, 024311 (2014)

[6] B. A. Brown, D. L. Fang, and M. Horoi, Phys. Rev. C 92, $041301(2015)$
[7] J. Engel and J. Menéndez, Rep. Prog. Phys. 80, 046301 (2017)

[8] L. Coraggio, A. Covello, A. Gargano, N. Itaco, and T. T. S. Kuo, Prog. Part. Nucl. Phys. 62, 135 (2009)

[9] L. Coraggio, A. Covello, A. Gargano, N. Itaco, and T. T. S. Kuo, Annals of Physics 327, 2125 (2012)

[10] L. Coraggio, L. De Angelis, T. Fukui, A. Gargano, and N. Itaco, Phys. Rev. C 95, 064324 (2017).

[11] L. Coraggio, L. De Angelis, T. Fukui, A. Gargano, and N. Itaco, in preparation.

[12] R. Machleidt, Phys. Rev. C 63, 024001 (2001)

[13] S. Bogner, T. T. S. Kuo, L. Coraggio, A. Covello, and N. Itaco, Phys. Rev. C 65, 051301(R) (2002)

[14] T. T. S. Kuo and E. Osnes, Lecture Notes in Physics, vol. 364 (Springer-Verlag, Berlin, 1990)

[15] H. M. Hoffmann, Y. Starkand, and M. W. Kirson, Nucl. Phys. A 266, 138 (1976)

[16] K. Suzuki and S. Y. Lee, Prog. Theor. Phys. 64, 2091 (1980)

[17] K. Suzuki and R. Okamoto, Prog. Theor. Phys. 93, 905 (1995)

[18] E. Caurier, G. Martinez-Pinedo, F. Nowacki, A. Poves, and A. P. Zuker, Rev. Mod. Phys. 77, 427 (2005)

[19] Data extracted using the NNDC On-line Data Service from the ENSDF database, file revised as of $\mathrm{Au}-$ gust 30, 2018.

[20] S. Mukhopadhyay, Phys Rev C 95, 014327 (2017)

[21] J. H. Thies et al., Phys. Rev. C 86, 014304 (2012)

[22] D. Frekers et al., Phys. Rev. C 94, 014614 (2016)

[23] P. Puppe et al., Phys. Rev. C 86, 044603 (2012)

[24] D. Frekers, P. Puppe, J. H. Thies, and H. Ejiri, Nucl. Phys. A 916, 219 (2013)

[25] A. S. Barabash, Nucl. Phys. A 935, 52 (2015)

[26] L. Coraggio, L. De Angelis, T. Fukui, A. Gargano, and $\mathrm{N}$. Itaco, in preparation.

[27] J. Menéndez, A. Poves, E. Caurier, F. Nowacki, Nucl. Phys. A 818, 139 (2009)

[28] R. A. Sen'kov and M. Horoi, Phys. Rev. C 90, 051301 (2014)

[29] R. A. Sen'kov, M. Horoi, and B. A. Brown, Phys. Rev. C 89, 054304 (2014) 